\title{
Fetomaternal Potassium Relations in the Rat on the Twentieth Day of Gestation. II. Effects of Maternal Hypokalemia
}

\author{
ALAN G. FANTEL \\ Central Laboratory for Human Embryology, Department of Pediatrics, School of Medicine, University of Washington, \\ Seattle, Washington, USA
}

\section{Summary}

Time-corrected measurements of transplacental and transamniotic electrical potentials were made in potassium-deficient rats on the 20th day of gestation. These averaged $6.4 \mathrm{mV}$ and $2.0 \mathrm{mV}$, respectively, in the deficient group as compared with 14.3 and 18.4, respectively, in the controls. The measured maternal potassium concentrations were $2.8 \mathrm{mEq} /$ liter in the $\mathrm{K}^{+}$-restricted animals and $4.8 \mathrm{mEq} /$ liter in the controls. These figures were substituted into the Nernst equation in order to predict fetal concentrations in plasma. Similar predictions were made for amniotic fluid assuming equilibrium with fetal plasma. Predicted concentrations in fetal plasma were $2.3 \mathrm{mEq} /$ liter in the deficient animals and 2.8 $\mathrm{mEq} /$ liter in the controls. By contrast, observed time-corrected potassium concentrations in fetal plasma were $4.0 \mathrm{mEq} /$ liter in deficient animals and $2.9 \mathrm{mEq} /$ liter in controls. Amniotic fluid values were predicted to be $4.7 \mathrm{mEq} / \mathrm{liter}$ in deficient and 2.5 $\mathrm{mEq} /$ liter in control animals. These values were compared with the observed averages in amniotic fluid of 4.7 and $4.6 \mathrm{mEq} /$ liter in the deficient and control groups, respectively.

\section{Speculation}

In chronic maternal hypokalemia, fetal potassium concentrations are maintained at a high level by active transfer, although diffusion along an electrochemical gradient is adequate to account for fetal levels in controls. Amniotic fluid concentrations are normally maintained by active transfer, probably across the fetal kidney. In maternal hypokalemia, active transfer of potassium across the fetal kidney is unnecessary to maintain values which are essentially unchanged from those found in control amniotic fluid.

In a previous report (7) a new method for the analysis of potassium concentrations in the plasma of late fetal rats was presented. It was shown that potassium concentrations are lower in fetal than in maternal plasma and that these concentrations are in electrochemical equilibrium maintained by passive transfer.

In order to predict fetal concentrations, the Nernst equation was rearranged and maternal plasma values (average 4.8 $\mathrm{mEq} /$ liter $)$ and time-corrected transplacental potentials $(14.3 \mathrm{mV}$, fetus positive) were substituted. It was found that the predicted value differed insignificantly from the observed concentrations, making it likely that potassium transfer to the late fetal rat occurs largely by diffusional processes. On the other hand, it was found that amniotic fluid concentrations significantly exceeded predicted levels and it was suggested that potassium was actively transferred into the amniotic fluid, probably across the fetal kidney.

The observed fetal concentrations presented were considerably lower than those of any previous study and it was suggested that these inconsistencies could be explained by artifactual elevations in potassium concentration in earlier work. Studies by Battaglia
(1) as well as Maniey (9) demonstrated the considerable susceptibility of fetal plasma to hypoxia and anesthesia-induced shifts of intracellular solutes, and efforts were made in this study to prevent as well as correct for these events. Fetal concentrations were shown to rise rapidly following intrauterine incision and this effect was corrected mathematically.

Because of the requirement for potassium as a major solute in cell mass increase, it might be expected that the induced maternal deficiency state would have teratogenic consequences, particularly if transport were strictly diffusional. This appears to be the case for organs grown in vitro as Crocker (3) and Crocker and Vernier (4) have shown that normal development of mouse and human kidneys require high potassium concentrations. Additionally, McDonald et al. (10) have shown that optimal rates of DNA synthesis in baby hamster kidney cells grown in vitro are dependent upon the maintenance of membrane potentials which require extracellular potassium concentrations in the range of $64 \mathrm{mM}$.

Despite these demonstrations, teratologic experiments performed in vivo have failed to correlate significant teratogenesis with maternal potassium deficiency. Ellison and Maren (6) found that maternal potassium restriction led only to occasionally absent or asymmetric ribs. A study of Gibson and Becker (8) showed some signs of growth retardation such as bradygnathia and missing or nonossification of vertebral bodies.

In their study, Dancis and Springer (5) showed that severe potassium depletion of pregnant rats by dietary restriction failed to greatly alter concentrations in fetal tissues. On the other hand, maternal hyperkalemia induced either by hyponatremia or $\mathrm{KCl}$ infusions led to fetal hyperkalemia. Unfortunately, control values presented in this paper suggested the intervention of artifactual hyperkalemia.

In a study performed by Seranno (12) in the pregnant bitch, it was again found that fetal concentrations in plasma or muscle failed to be significantly influenced by great reductions in maternal serum concentrations of potassium. Additionally, no effects on pregnancy outcome, litter size, amniotic fluid concentrations, or fetal weights were seen.

As a result of the above noted observations, a study of potassium homeostatis during maternal hypokalemia was undertaken.

\section{MATERIALS AND METHODS}

Sprague-Dawley females were maintained at the colony of the Central Laboratory for Human Embryology at the University of Washington where a 14-hr dark/10-hr light cycle was employed. Females were checked nightly for estrus signs according to the method of Blandau et al. (2). If positive they were mated overnight and the cages were checked in the morning for the presence of copulation plugs. If plugs were found the animals were considered to be in day 0 of pregnancy.

All animals had food and water ad libitum with the exception of the experimental animals which on a day between days 12 and 
14 were placed on a potassium-free test diet plus distilled water (Nutritional Biochemical) for the duration of pregnancy. According to the manufacturer, this diet contains "nil to trace amounts of potassium."

The methodology employed for the measurement of transplacental potentials was adapted from Mellor (11) and has been described in detail (7). Fetuses were sampled serially and potentials were measured between the maternal peritoneal fluid and the fetal amniotic fluid or the fetal peritoneal fluid. Because potentials were recorded serially it was possible to correct mathematically for temporal changes.

The procedure for sampling of plasma has also been described in detail (7). It involved taking blood samples from the umbilical artery or vein of individual fetuses by micropuncture of the desired vessel. The measurement of potassium concentration was carried out by flame photometry (Philips-Unicam SP60A) of appropriately diluted $10-\mu 1$ plasma samples. Maternal blood was sampled in a manner which was identical to fetal blood, but collection was from a superficial artery in the thigh. As with potential measurements, sampling was done serially and careful notation of time following uterine incision was made.

The rewritten Nernst equation is presented below

$$
\left[\mathrm{K}^{+}\right]_{0}=\frac{\left[\mathrm{K}^{+}\right]_{\mathrm{i}}}{\mathrm{e}^{\mathrm{x}}\left[\log _{\mathrm{e}} 10 \mathrm{E}_{\mathrm{K}} / 62.5\right]}
$$

where: $\left[\mathrm{K}^{+}\right]_{0}$ represents the potassium ion concentration in the fetal plasma or amniotic fluid; $\left[\mathrm{K}^{+}\right]_{i}$ represents the potassium concentration in the maternal or fetal plasma; $E_{K}$ represents the average potential across the membrane under consideration.

In a steady state situation, the rearranged Nernst equation permits the distinction between active and passive transfers of ions across membranes. If passive transfer determines concentration relations, the observed values on the fetal side (outside) of the membrane should be similar to those which have been predicted by the rearranged equation. The same procedure was followed for the analysis of the amniotic fluid concentrations. Amniotic fluid was assumed to be in electrochemical balance with the fetal rather than the maternal plasma, although the predicted values are similar using either source for $\left[\mathrm{K}^{+}\right]_{\mathrm{i}}$.

\section{RESULTS}

There was considerable scatter in both the transplacental and transamniotic electrical potentials of treated and control groups. The values appeared to become increasingly positive in time for the transplacental potentials of the experimental group whereas they decline over the same time in the controls. When they were drawn back to the time of the initial uterine incision ("zero time") they averaged $6.4 \mathrm{mV}$ positive to the maternal peritoneum in the deficient group and $14.3 \mathrm{mV}$ positive in the controls. Transamniotic values showed a steep rise over time in the deficient group with zero time corrected values being $+2.0 \mathrm{mV}$. For the control, amniotic fluid values fell over time and projected back to zero time at $+18.4 \mathrm{mV}$.

The maternal plasma concentrations differed significantly between the two groups. The values were $2.8 \pm 0.1 \mathrm{mEq} / \mathrm{liter}$ (mean and SE) in the deficient group and $4.8 \pm 0.2 \mathrm{mEq} / \mathrm{liter}$ in the controls. With a total of 8 deficient and 10 control animals, using Student's $t$-test $P<0.001$. These values remained stable throughout the period of the experiment.

Based upon the observed and time-corrected potential differences and the maternal plasma concentrations, the following expected values were calculated using the assumptions implicit in the Nernst relationship. If concentrations in fetal plasma were in electrochemical equilibrium with those in the maternal plasma, the expected values for the experimental group would be $2.3 \pm 0.7$ $\mathrm{mEq} /$ liter (mean and 95\% confidence interval) and $2.8 \pm 0.3$ in the controls.

Figure 1 presents the observed concentrations in fetal plasma along with the confidence intervals at the y intercept. These yintercept values represent the zero time concentrations. It can be

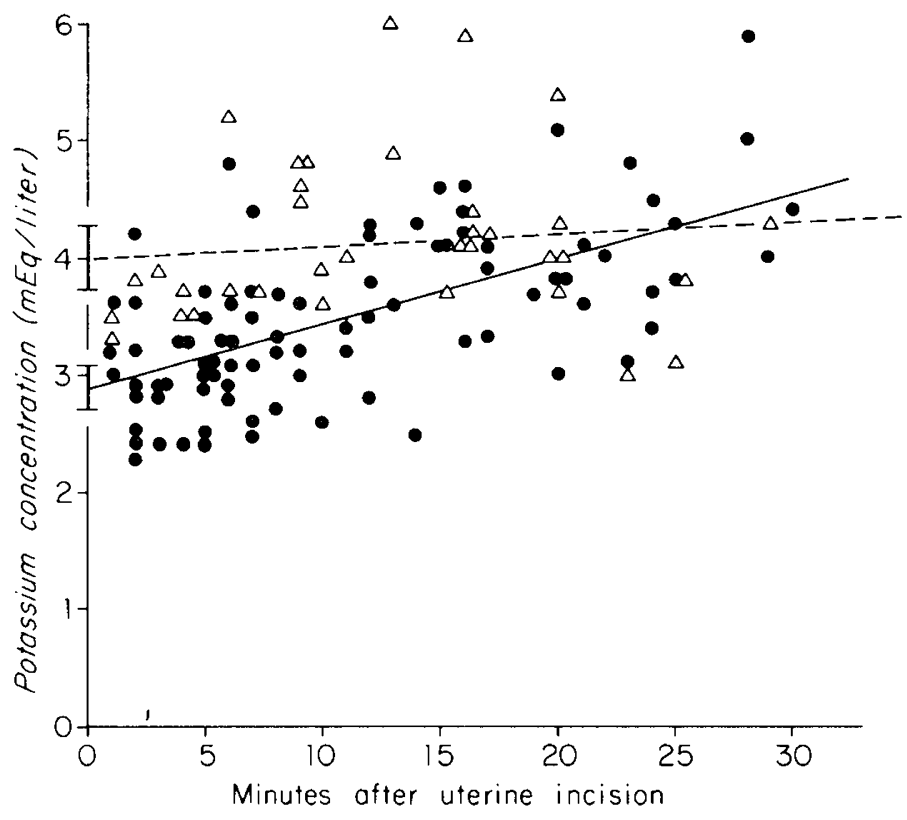

Fig. 1. Comparison of potassium concentrations in fetal plasma of potassium-restricted and control animals. $\Delta$, deficient animals; $\boldsymbol{\bullet}$, control animals. - - - , regression of deficient animals; - - , regression of control animals. The bars at the $y$ intercepts represent the $99 \%$ confidence intervals for the respective regressions. Thirty-five plasma samples were obtained from 7 litters of deficient animals and 89 samples (from 64 fetuses) from 15 litters of controls.

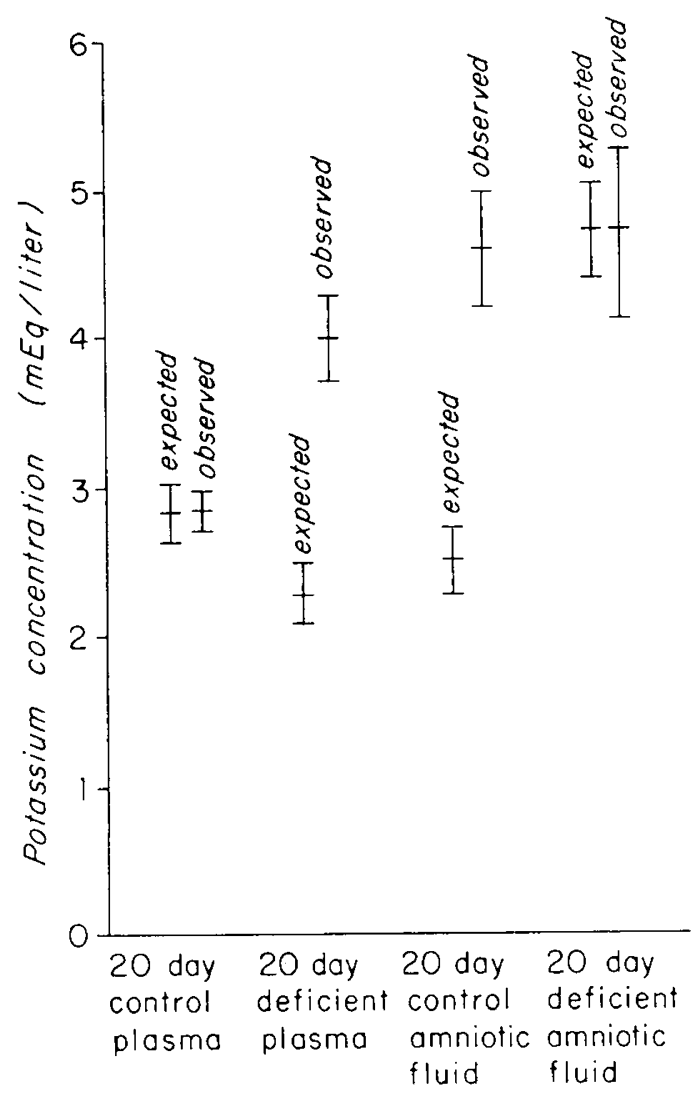

Fig. 2. Comparison of expected and observed time-corrected concentrations of potassium in fetal plasma and amniotic fluid. The brackets indicate the $99 \%$ confidence intervals about the mean or $y$ intercept. Transplacental potentials were determined from 23 deficient and 42 control fetuses. Transamniotic potentials were recorded from 21 deficient and 47 control fetuses. 
seen that the fetuses from the deficient mothers were significantly higher than the controls ( 4.0 vs. $2.9 \mathrm{mEq} / \mathrm{liter}$ ). As can be seen from Figure 2, the observed values in the treated group were significantly higher than the predicted values $(4.0$ vs. 2.3 $\mathrm{mEq} /$ liter) although there was no significant difference between the observed and expected concentrations in the controls (2.9 vs. $2.8 \mathrm{mEq} / \mathrm{liter}$ ).

In order to compare the two groups in a different fashion, Nernst predictions of fetal concentration were prepared for each minute of experiment time employing the constant maternal concentrations and the changing potentials for each group. The observed values at each minute of experimental time were subtracted from the predicted values for that same time and the mean difference for each group was calculated. The mean difference for the deficient group was $+2.1 \pm 0.1 \mathrm{mEq} /$ liter vs. $+0.5 \pm 0.1$ $\mathrm{mEq} /$ liter in the controls. The Student's $t$ value for the difference between the mean differences is 11.8 which has a $P<0.001$. Therefore, the two groups differ significantly in their deviation from values predicted by the Nernst equilibrium conditions.

Potassium concentrations in amniotic fluid were slightly but not significantly higher in the deficient group than in the controls (4.7 $\pm 0.2 v s .4 .6 \pm 0.1 \mathrm{mEq} /$ liter, respectively). In the deficient group, the predicted and the observed values were indistinguishable, as can be seen in Figure 2. This relationship contrasts with that in the control fetuses where the predicted value was $2.5 \mathrm{mEq} / \mathrm{liter}$, significantly below the observed concentration. The predicted values employed fetal plasma concentrations and the derived potential difference between fetal plasma and amniotic fluid, based upon the assumption that this was the site of major equilibrium. However, if amniotic fluid were assumed to be in electrochemical equilibrium with maternal plasma, the predicted values would show little change in the controls and a decrease to 2.6 $\mathrm{mEq} /$ liter in the deficient animals.

\section{DISCUSSION}

Maternal potassium restriction leads to a decline in the electropositivity of the fetal side of the placenta in the day 20 rat. The expected result of the demonstrated average $42 \%$ fall in potassium concentration in maternal plasma would be a slight reduction in the fetal plasma levels in the Nernst equilibrium model. The fact that the actual concentrations in the experimental fetuses significantly exceeded those predicted suggests that in chronic maternal hypokalemia, active transfer of potassium into fetal plasma occurs. This is in clear distinction to the controls where expected and observed values coincide closely and active transfer need not be postulated. The significant difference between the mean differences of the two groups reinforces the conclusion that they behave in dissimilar fashion as a result of maternal potassium restriction in the treated animals.

Potassium concentrations in amniotic fluid significantly exceed predictions in the control fetuses irrespective of the assumed site of equilibration. Although the concentrations in amniotic fluid of deficient animals do not differ appreciably from those of controls they do not appear to require active transfer. It is likely, however, that active transfer of potassium occurs in the normokalemic state and it is suggested that the site of this transfer is the fetal kidney. This would reinforce the central role of fetal urine in determining the chemical composition of the amniotic fluid.

\section{SUMMARY}

In the normal day 20 fetal rat, potassium concentrations in plasma appear to be in electrochemical equilibrium with those of the mother. On the other hand, in chronic maternal hypokalemia, fetal concentrations are significantly higher than those derived from predictions of passive transfer and active transfer is therefore postulated. Potassium concentrations in amniotic fluid appear to be relatively uninfluenced by maternal potassium status and it is suggested that these concentrations are normally derived by active transfer into the fluid pool. In the hypokalemic pregnant rat, however, active transfer is not required to maintain amniotic fluid concentrations.

\section{REFERENCES AND NOTES}

1. Battaglia, F. C., Meschia, G., Hellegers, A., and Barron, D. H.: The effects of acute hypoxia on the osmotic pressure of the plasma. Q. J. Exp Physiol., 43: 197 (1958).

2. Blandau, R. J., Boling, J. L., and Young, W. C.: The length of heat in the albino rat as determined by a copulatory response. Anat. Rec., 79: 453 (1941).

3. Crocker, J. F. S.: Human enbryonic kidneys in organ culture: Abnormalities of development induced by decreased potassium. Science, 181: 1178 (1973)

4. Crocker, J. F. S., and Vernier, R. L.: Fetal kidney in organ culture: Abnormalities of development induced by decreased amounts of potassium. Science, 169: 485 (1970).

5. Dancis, J., and Springer, D.: Fetal homeostasis in maternal malnutrition: Potassium and sodium deficiency in rats. Pediat. Res., 4: 345 (1970).

6. Ellison, A. C., and Maren, T. H.: The effects of metabolic alterations on teratogenesis. Johns Hopkins Med. J., 130: 87 (1972)

7. Fantel, A. G.: Fetomaternal potassium relations in the fetal rat on the twentieth day of gestation. Pediat. Res., 9: 527 (1975).

8. Gibson, J. E. and Becker, B. A.: Placental transfer, embryotoxicity and teratogenicity of thallium sulfate in normal and potassium-deficient rats. Toxicol. Appl. Pharmacol., 16: $120(1970)$

9. Maniey, J.: Teneur en potassium du plasma du foetus de rat: Influence des conditions de prelevement du sang. C. R. Soc. Biol., 153: 1731 (1959).

10. McDonald, T. F., Sachs, H. G., Orr, C. W., and Ebert, J. D.: External potassium and baby hamster kidney cells: Intracellular ions, ATP, growth, DNA synthesis and membrane potential. Dev. Biol., 23: 290 (1972).

11. Mellor, D. F.: Potential differences between mother and foetus at different gestational ages in the rat, rabbit and guinea-pig. J. Physiol. (London), 204. 395 (1969).

12. Seranno, C. V.: Potassium deficiency in the pregnant dog. Ph.D. dissertation, University of North Carolina (1963).

13. Stewart, E. L. and Welt, L. G.: Protection of the fetus in experimental potassium depletion. Amer. J. Physiol., 200: 824 (1961).

14. The author wishes to express his appreciation for the assistance of Dr. Thomas H. Shepard, Ms. Glenda Schneider, and Ms. Barbara Brownfield.

15. This research was supported by National Institutes of Health Grants HD00836, DE02918, and HD00180.

16. Requests for reprints should be addressed to: Dr. A. G. Fantel, Central Laboratory for Human Enbryology, Department of Pediatrics, RD-20, University of Washington, Seattle, WA 98195 (USA)

17. Received for publication September 30, 1977

18. Accepted for publication December 14, 1977. 